\title{
LA SUBORDONNÉE CONDITIONNELLE DANS LE BAS LATIN, LE REGISTRE POPULAIRE
}

Nous commençons ce travail par dire qu'on rencontre des éléments de latin populaire à l'époque tardive ${ }^{1}$ non seulement dans des inscriptions ou des textes de médecine vétérinaire, ou bien d'art gastronomique, rédigés par des gens sans instruction, mais encore dans les écrits des ecclésiastiques, surtout à la suite de recommandations telles que:

- "melius est reprehendant nos grammatici quam non intellegant populi.", saint Augustin, In ps., 138, 20, à la traduction:

"Il vaut mieux que les grammairiens nous réprimandent plutôt que les populations ne nous comprennent pas."

ou: - "... philosophantem rhetorem intellegunt pauci, loquentem rusticum multi.", Grégoire de Tours, Hist. Franc., Proem.

Des éléments de latin populaire apparaissent parfois chez divers auteurs cultivés, soit qu'ils y mettent trop de hâte (donc, de manière involontaire), soit alors qu'ils reproduisent le langage des ignorants. D'autres fois les éléments de latin populaire sont adoptés par les auteurs cultivés pour des raisons stylistiques, ou pour des raisons de métrique.

\section{L'inventaire des conjonctions et des locutions conjonctives à l'époque tardive, dans le registre populaire.}

À la basse époque, la conjonction si est extrêmement fréquente, autant dans le registre populaire que dans le registre cultivé ${ }^{2}$. Voici un fragment de Lex Salica:

"Si quis coccum aut gallina furauerit, .... culpabilis iudicetur.", 7, 63 .

\footnotetext{
${ }^{1} \mathrm{Par}$ "époque tardive" nous comprenons la période allant du début du IIIe siècle p. Chr. (ou la fin du IIe siècle p. Chr.) aux premières décennies du VIIIe siècle, autant que le latin était encore une langue vivante.

${ }^{2}$ Quant aux raisons de la survivance de la conjonction si tout le long de la latinité vivante, voir R. Iordache, Remarques sur les raisons de la conservation de la conjonction latine 'si' dans les langues romanes, dans "Linguistica", no. XXVIII, Ljubljana, 1988, pp. 35-46.

3 Texte cité d'après l'édition de H. Geffcken, Leipzig, 1898.

On remarque, dans le passage ci-dessus, l'accusatif singulier gallina à l'omission de la - $m$ finale et l'emploi de la forme active, populaire, furauerit, à la place de la forme moyenne de verbe déponent.On observe également l'emploi, à la suite d'une confusion, du substantif coccum (au redoublement populaire -cc) au lieu de gallum (nom. gallus).
} 
En latin populaire, la conjonction si apparaît assez souvent en corrélation avec les adverbes sic, iam, tunc, le pronom hoc, ou les locutions ob hoc, hoc modo (voir, par exemple, Mulomedicina Chironis, 4, 46, 399; ibid., 5, 56, 516; ibid., 6, 32, 562, etc. ).

En tant que corrélatifs on rencontre aussi certe et saltem (voir saint Cassien, Inst. coenob.. 19,6 etc. ).

Chez les auteurs cultivés, la corrélation habituelle est: "si-ita". Voici un passage de saint Grégoire le Grand:

"Quem tamen ita suscipiendum esse cognosce, si et uxor ipsius similiter conuerti uoluerit.", Reg., 6, 47 (I, p. 422, 25) ${ }^{4}$.

Dans les textes écrits aux VIe et VIle siècles, mais aussi plus tard - aux VIIIe et IXe siècles (quand le latin avait cessé d'être une langue vivante), on remarque, pour la conjonction, la graphie se - voir, par exemple, Formulae Andecauenses, A, $11^{5}$.

\section{Composés de la conjonction 'si'}

Quod si et quodsi (en un seul mot), pour réaliser la liaison entre des phrases complexes sont spécifiques du latin cultivé (voir saint Augustin, Catech., lo; ibid., 11, etc.). La locution aussi bien que le composé quodsi sont, parfois, attestés dans des textes écrits en latin populaire. Voici un passage de Pseudo-Hyginus:

"Quodsi cohortes praetoriae impares acceptae fuerint, ....

loco cohortis equites praetoriani ponentur.", Met. castr., $8{ }^{6}$.

Pour 1'emploi de quodsi, voir également Pseudo-Hyginus, Met. castr., 41; Testamentum porcelli, 3; Palladius, Agr., 5, 7, etc. (les textes sont indiqués dans l'ordre chronologique de leur rédaction).

L'emploi de siue, à la variante seu, se développe continûment dans le latin populaire de la basse époque. Siue apparaît seul, au sens de "ou", pour réaliser la disjonction entre deux éléments de proposition (voir Pseudo-Hyginus, Met. castr., 12; ibid., 50, etc.) ou entre deux propositions de la même catégorie (voir saint Isidore de Seville, Et., 1, 7, 2, etc.).

La corrélation "siue - siue" (ou: "seu - seu") est également fréquente dans le registre populaire tardif, en réalisant la coordination disjonctive entre des éléments de proposition ou entre des propositions (voir, par exemple, Marcellus Empiricus, Med., 16,58; ibid., 28,16; Victor Vitensis, Hist. Persec., 3, 47, etc.). Pour des raisons stylistiques, dans de longues suites, siue est parfois utilisé par quatre fois, quelquefois par six ou sept fois (voir saint Augustin, Anim., 32, 3; Salvien, Gub. D., 4, 10-p. 66, 1.14-16).

\footnotetext{
${ }^{4}$ Exemple emprunté à D. Norberg, In 'Registrum' Gregorii Magni studia critica, Uppsala - Leipzig, 1939, II, p. 145 .

5 Texte cité d'après I. Iordan (coordonateur), Crestomathie romane, I, Bucarest, 1962 (l'édition de K. Zeumer, Formulae merovingici et karolini aevi, Legum sectio V, dans "M. G. h.", Hanovre, 1886).

${ }^{6}$ Selon l'édition d'A. Grillone, Hygini qui dicitur de metatione castrorum liber, Teubner, 1977.
} 
Pour la disjonction entre deux ou plusieurs propositions conditionnelles, le latin populaire emploie également aut, ou aut si (voir Lex Salica, 8, 2; ibid., 12, 3; ibid., 17, 2, etc.; Edictus Rothari, 4; ibid., 169, etc. ${ }^{7}$ ).

Chez les auteurs cultivés, les propositions conditionnelles enchaînées par disjonction sont d'habitude construites à l'optatif (voir Claudien Mamert, Stat. an., 2, 5-p. 118, 1.1 sqq; saint Avit, 6, 380 ${ }^{8}$, etc.). Dans les textes écrits en latin populaire, qu'il s'agisse de la disjonction des conditionnelles, ou des concessives, l'indicatif prédomine (voir, par exemple, Peregrinatio Egeriae, 37, 4, etc.).

En ce qui concerne les conditionnelles négatives, nisi continue d'apparaître dans les textes influencés par le latin populaire (voir Victor Vitensis, Hist. persec., 1, 27; Jordanès, Get., 173; ibid., 210,etc.). Nisi continue également d'être utilisé pour régir le complément d'exception et la subordonnée correspondante, autant dans le registre populaire, que dans le registre cultivé ${ }^{9}$. Parallèlement, on observe l'extension de l'emploi de la séquence 'si non' tant pour les conditionnelles négatives, que pour le complément et la subordonnée exceptifs.

\section{Locutions conjonctives fondées sur 'si'}

À l'époque tardive sont employées les locutions suivantes: si forte, si quidem, sane si, plane si, si modo, si tantum, si tamen, si uero, si contra, e contra si, sed si.

Si tantum et si tamen remplacent dans le latin populaire tardif la locution si modo. En témoigne le passage suivant:

"si tantum eos inopes esse cupitis, concedite id ipsorum deuotioni.", Salvien, Eccl., $3,24$.

Voir également Jordanès, Rom., $126^{10}$.

Pour si uero, voir un passage de Vitae Patrum:

"... si autem dixero: 'Da eam (sc.: hereditatem) parentibus tuis', non est tibi merces; si uero dicam: 'Da pauperibus', securus eris.", $5,10,56$.

Pour la locution si uero, voir aussi Serenus Sammonicus, Med., 52, v. $956^{11}$. Pour si quis uero, voir Formulae merouingici et karolini aeui, Legum sectio $V$, Formulae Andecauenses, B, 19.

Saint Avit emploie la locution: e contra si (p. 30, 1. 30); plus fréquente est la locution si contra (voir Ulpien, Dig., 11, 7, 14, 15; Paulinus Nolanus, Carm., 10, 331, etc.)

${ }^{7}$ Edictus Rothari est cité d'après I. Iordan, Crestomathie romane, I, op. cit. (l'édition de Fr. Bluhme, Edictus Langobardorum, Legum tomus IV, dans "M. G. h.", Hanovre, 1868).

${ }^{8}$ L'exemple de saint Avit est tiré de H. Goelzer, Le latin de saint Avit, Paris, 1909, p. 359 (l'édition de R. Peiper, dans "M. G. h.", VI -2).

${ }^{9}$ Pour ce qui est de la fréquence de l'emploi de nisi à l'époque tardive, pour introduire le complément d'exception et la subordonnée correspondante, voir $\mathrm{R}$. Iordache, Remarques concernant l'histoire des subordonnées d'exception en latin et dans les langues romanes, dans "Linguistica", vol. XL / 1, Ljubljana, 2000, p. 21; ibid., pp. 25-6.

${ }^{10}$ La locution si tantum apparaît déjà chez Curtius Rufus, Hist. Alex. M., 3, 6, 3.

11 Dans l'édition de Fried. Vollmer, Corpus medicorum latinorum, II - 3, Leipzig, Teubner, 1916. 
Conjonctions non-spécifiques pour introduire des subordonnées conditionnelles dans le registre populaire

Quando construit à l'indicatif est fréquent chez les auteurs tardifs. Voici un passage de Salvien:

"Et quae esse, rogo, Romano statui spes potest, quando castiores ac puriores barbari quam Romani sunt?", Gub. D., 7, 107 (p. 190, 1. 32 - p. 191, 1. 1-2).

$V b i$ et $d u m$ apparaissent également dans des contextes temporels- conditionnels:

- "Nam quae, rogo, spes esset capto, ubi indulgi uel filio nefas habebatur?", Jordanès, Get., 57.

- "nihil refert integram abire corporis nauem, an dissipatam, dum animae nauigatio euertatur.", Tertullien, An., 52.

Pour l'emploi de dum, voir aussi Ulpien, Dig., 39, 3, 1, 11, etc. ${ }^{12}$.

Dans des contextes de manière-conditionnels, on emploie la conjonction ut (spécifique du latin cultivé) et aussi quantum et la locution in quantum (propres au latin populaire). Voici quelques occurrences de saint Grégoire le Grand:

- "sanctitas uestra ei, ut potuerit, .... opem ferre studeat.", Reg., 9, 127 (II, p. 127, 28);

- "fraternitas uestra ei se, in quantum ualet, impendat.", Reg., 9, 127 (II, p. 127, 31) ${ }^{13}$.

Ces formules nous rappellent les séquences d'ablatif absolu fréquentes dans le latin ecclésiastique: "Deo fauente", "Christo iuuante", "spiritu sancto praestante".

La locution 'in quantum' est souvent attestée à sens conditionnel aux époques postclassique et tardive. Voici un passage de Tacite:

"Qui nationem eam regebant, in quantum Germani regnantur.", Ann. 13, 54, 1.

Dans de tels passages, la locution in quantum a le sens: "seulement dans la mesure où", "si".

Voici des citations d'auteurs tardifs:

- "Castra, in quantum fieri potuerit, tertiata esse debebunt ....", Pseudo-Hyginus, 21.

- "Omnia uera sunt, in quantum sunt.", saint Augustin, Conf., 7, 15, 21.

À voir également Tertullien, Apol., 38, 4; Pseudo-Hyginus, 2; Orose, Adu. Pagan., $7,6,11$ etc.

Une autre locution qui est utilisée à sens conditionnel dans le registre populaire des époques postclassique et tardive c'est 'in quo'. Exempli gratia:

- " ... ad nos apostolus clamat: 'in quo ... alium iudicas, te ipsum condemnas; eadem enim agis quae iudicas.' ....", Salvien, Gub. D., 4, 12.

12 Pour d'autres exemples de dum à sens conditionnel, voir Thesaurus linguae Latinae, V - 1, Leipzig, Teubner, 1934 , p. $2216,1.72$ sqq..

13 Les citations de saint Grégoire le Grand sont empruntées à D. Norberg, In 'Registrum' Gregorii Magni studia critica, op. cit., vol. II, p. 212. 
- Voir également Itala, Luc., 12, 32; Victor Vitensis, Hist. persec., 2, 92.14

Conf. l'emploi de la locution "in o quid" à sens conditionnel dans le premier texte gallo-roman "Les Serments de Strasbourg", 3, 1, 1.

Nous faisons également remarquer que les auteurs tardifs utilisent parfois des subordonnées comparatives de progression s'ajoutant un sens conditionnel. Preuve les passages suivants:

- "Quo plus te operari uiderit populus, magis diliget.", saint Ambroise, Off. ministr., 2, 16, 78.

- "Consilii autem operisque collatio, quo in plures diffunditur, eo radundantior manet et in suum fontem recurrit.", saint Ambroise, Off. ministr., 2, 15, 75.

Dans les textes écrits en latin populaire, on remarque l'accumulation de conjonctions ou de conjonctions et locutions à sens conditionnel. Voici un passage de Gregorius Magnus:

- "In quantum uero ut praeualet fraternitas tua, opponere se pro pauperibus, pro oppressis debet.", Reg., 10, 15 (II, p. 250, 3) ${ }^{15}$.

Les auteurs cultivés de l'epoque tardive utilisent cum et quatenus à sens conditionnel. Ils y ajoutent, bien sûr, l'emploi de si, de ses composés et ses locutions.

La formule: "quae (haec) cum ita sint" apparaît, parfois, dans contexte conditionnel, voir Victor Vitensis, Hist. persec., 2, 95.

La négation pour les subordonnées régies par les conjonctions et les locutions non-spécifiques de l'idée de condition est toujours non, qu'il s'agisse du registre populaire, ou du registre cultivé.

\section{Conjonctions et locutions pour le sens "pourvu que".}

A. De la série ancienne (dum, dummodo, modo, si modo) les auteurs de la basse epoque continuent d'employer dum et dummodo, qu'il s'agisse des auteurs cultivés ou de ceux influencés par le latin populaire.

Voici quelques exemples de dummodo utilisée dans des conditionnelles affirmatives et négatives:

- "Nam in tantum eum spernis, in tantum despicis, ut nec tibimet ipsi consulas, dummodo eius iussa contemnas.", Salvien, Eccl., 3, 82 (p. 296, 1. 14-15).

- Pour dummodo employée dans une conditionnelle affirmative, voir aussi saint Avit, p. 72,4 etc.

- Sed fratres meos honorare per omnia cupio sic que studeo honore singulos subuehi, dummodo non sit quod alteri iure ab altero possit opponi.", saint Grégoire le Grand, Reg., 2, 52 (I, p. 156, 40).

\footnotetext{
14 La locution in quo est, parfois, attestée chez les auteurs tardifs à sens causal (voir Victor Vitensis, Hist. persec., 2, 92). D'autres fois in quo apparaît à sens concessif, ou de manière (voir Thesaurus linguae Latinae, VII -1 , Leipzig, Teubner, 1938, p. 779, 1. 22 sqq.).

15 Exemple cité chez D. Norberg, In 'Registrum' Gregorii Magni studia critica, op. cit., vol. II, p. 212.
} 
Pour la corrélation "ita - dummodo nec .... nec", voir saint Grégoire le Grand, Reg., 4, 35 (I, p. 271,14) ${ }^{16}$. Chez certains auteurs tardifs on constate une accumulation de conjonctions, par exemple: "dummodo ut" suivie du subjonctif (Salvien, Eccl., 3, 82, p. 296, 1. 11-12).

B. La nouvelle série de locutions est représentée par tantum ut et tantum ne / non. On retrouve aussi l'emploi de tantum en qualité de conjonction.

Rappelons que chez Cicéron est attestée la locution tantummodo ut (Fam., 16, 12, 4), chez Ovide - la variante négative tantum ne (Met., 9, 21) ${ }^{17}$. Chez Sénèque apparaît tantum seule à la fonction de dummodo (voir Consol. ad Hel., 5; Ep., 78,25).

Pour l'utilisation de la locution tantum ut à l'époque tardive, voir Commodien, Instr., 2, 18, 16; Paulinus Petricordiae, Mart., 2, $9^{18}$, etc.

Pour tantum ne et tantum non (ce syntagme à la place de tantum ne), voir les passages suivants:

- "... tantum a ueritate rerum non aberret animus, facile est ut .... ex ipsa occasione discat ...", saint Augustin, Catech., 11 (16), 1.19

- "nouum hoc monstri genus est cuilibet ...., tantum ne sibi.", Salvien, Eccl., 3, 12 (p. 272, 1. 22-24).

La locution tantum si et la séquence tantum est si au sens de "tantum si" sont rares. Voici un passage de Langobardische Gesetze:

"Tantum est si ancilla aut filii simul cum patre ad furtum faciendum non ambolauerint; nam si fecerint, similis illi efficiantur.", 26120.

Les auteurs tardifs emploient aussi tantum en qualité de conjonction. La transformation des adverbes en conjonctions de subordination est un phénomène fréquent à la basse époque ${ }^{21}$. Voici une citation d'Orose:

"Consolentur se 22 gentiles, in quantum uolunt, Iudaeorum haereticorumque suppliciis, tantum et unum Deum esse et eundem personarum acceptorem non esse .... fateantur.", Adu. Pagan., 7, 33, 18.

16 Exemple emprunté à D. Norberg, In 'Registrum' Gregorii Magni studia critica, op. cit., vol. II, p. 145.

17 Exemples tirés de R. Kühner - C. Stegmann - A. Thierfelder, Ausführliche Grammatik der lateinischen Sprache, Hanovie, 1971, vol. II -1, p. $183^{3}$.

${ }^{18}$ Exemple cité d'après "C. S. E. L.", vol. XVI, Vienne, 1888, p. 35.

19 Exemple tiré d’A. Blaise, Manuel du latin chrétien, Strasbourg, 1955, p. 169. Dans la citation ci-dessus, on observe aussi le remplacement de l'Accusatif + l'Infinitif (en dépendance de l'expression "facile est") par la formule "ut + le subjonctif".

${ }^{20}$ Exemple présenté par B. Löfstedt, Studien über die Sprache der langobardischen Gesetze, Stockholm, 1961, p. 263. Dans le passage ci-dessus, on observe ambolauerint à la place de ambulauerint; similis au lieu de similes.

21 Pour la création de conjonctions de subordination à partir d'adverbes, de locutions adverbiales et de prépositions, voir J. Herman, La formation du système roman des conjonctions de subordination, Berlin, Akademie Verlag, 1963, pp. 117-119; voir aussi R. Iordache, Exprimarea ideii de concesie in limba latina; Bucuresti, Maison éd. "Paideia-Academica", 2002, p.115; R. Iordache, Remarques sur la subordonnée temporelle à l'époque classique et à l'époque tardive, chez Jordanès, dans "Linguistica", vol. XXXIII, Ljubljana, 1993, p. 77 sqq..

$22 \mathrm{Se}$ - superflu dans ce passage. 
C. L'utilisation de locutions telles que: sub hac condicione, cum hac condicione, ou cum hoc, in hoc, suivies de propositions à fonction d'apposition, introduites par ut / quod, ou si. Exempli gratia:

- " ... sub illa $(m)$ condicionem, ut hoc ... non bioletur sepulcrum.", Inscriptiones Christianae - Rossi, no. $1125^{23}$.

- "si puella ... ad .... ingenuum uenerit in ea condicione, ut eum sibi maritum adquirat.", Lex Visigotharum, 3, 2, 824.

Vers la fin de la latinité vivante, les locutions du type $B$ et aussi $C$ se substituent presque complètement aux conjonctions et locutions anciennes au sens de "pourvu que".

\section{Dérogations aux normes du latin cultivé quant à l'emploi des modes.}

\section{L'extension de l'emploi de l'indicatif à la place de l'optatif:}

L'emploi de l'indicatif à la place de l'optatif est un phénomène ancien, présent à l'époque préclassique ${ }^{25}$. Aux époques suivantes, surtout dans le registre populaire, ce phénomène est de plus en plus fréquent.

L'emploi de l'indicatif au lieu de l'optatif a lieu tantôt en subordonnée, tantôt en régissante, parfois - dans les deux propositions de la période conditionnelle. Voici un passage où l'indicatif présent (pour condition réalisable dans le présent) a remplacé l'optatif présent autant dans la subordonnée que dans la régissante:

"Si enim extra conscientiam suam sunt quaecumque dico, nequaquam ad iniuriam eius spectant cuncta quae dico.", Salvien, Gub. D., 4, 17. ${ }^{26}$

Également fréquent est l'emploi de l'indicatif pour: a) le raport d'irréalité dans le présent / futur, et b) le rapport d'irréalité dans le passé. Ce phénomène, présent à toutes les époques du latin vivant, est attesté dans la subordonnée, aussi bien que dans la régissante:

Voici quelques exemples:

a) "Nam cum his multo melius agebatur, si non compellerentur hoc uelle.", Salvien, Gub. D., 5, 27 (p.110, 1. 22-23).

${ }^{23}$ Exemple tiré de Thesaurus linguae Latinae, IV, Teubner, 1909, p. 129, 1. 29-30. Ce passage renferme toute sorte d'erreurs: la préposition sub accompagnée de l'accusatif; illa $(m)$ utilisé àla place de $e a m ; u t . . . n o n$, au lieu de ne; bioletur, àla place de uioletur.

${ }^{24}$ Exemple emprunté à Thesaurus linguae Latinae, IV, op.cit., p. 129, 1. 37 sqq.. Pour d'autres détails concernant le complément de condition à l'époque tardive, voir $\mathrm{R}$. Iordache, Le complément hypothétique en latin, dans "Linguistica", vol. 42, Ljubljana, 2002, pp. 25-26.

25 Voir Ch. E. Bennett, Syntax of early Latin, vol. I, Boston, 1910, p. 60 sqq.; ibid., I, p. 274-77; ibid., I, p. 278; ibid., p. 280; ibid., p. 282. Nous nous rapportons aux conditionnelles courantes, et non pas àla catégorie de conditionnelles introduites par dum, dummodo, modo (au sens de: "pourvu que"). Voir aussi R. Iordache, Exprimarea ideii de conditie in limba latina, Bucuresti, "Bren", 2003, p. 89; ibid, pp. 90-93; ibid., p. 108; ibid., pp. 120-122.

${ }^{26}$ Le passage est discuté dans l'Index uerborum et locutionum de l'édition de Fr. Pauly, Salviani presbyteri Massiliensis opera omnia, dans "C. S. E. L.", vol. VIII, Vienne, 1883. 
b) “... tantum uirum persona penitus non grauauerat, nisi causa multasset.", Salvien, Gub. D., 2, 15 (p.36, 1. 13-14);

- "cuius amore et desiderio populus .... potuerat membra digni corporis rapere, nisi consilio prudenti .... nesciente multitudine sepeliretur.", Victor Vitensis, Hist. persec., 1, 27 (p. 13, 1. 3-6).

- Voir aussi saint Avit, p. 129, 12 et vol. 5, 144.

H. Goelzer soulignait dans sa Monographie consacrée à saint Avit "qu'à partir d'Ammien Marcellin ... on voit n'importe quel verbe exprimer l'irréel du passé au plus-que-parfait de l'indicatif." ${ }^{27}$.

Pour ce qui est de l'expression de l'irréalité dans le passé par le parfait de l'indicatif, voir Jordanès:

"nisi paruisset, ... fecit audaciam.", Rom., 168.

Tout aussi fréquente est l'expression du potentiel par le futur simple, souvent en corrélation, dans les textes ecclésiastiques, avec l'optatif présent. Par exemple:

"Tunc enim ualebo quae desiderata sunt indicare, si ipsi dignentur pro me misero domino supplicare.", Passio septem monachorum, $1^{28}$.

\section{Confusion entre l'expression du potentiel et de l'irréel.}

La confusion entre l'expression du potentiel et l'expression de l'irréel, existante déjà à l'époque préclassique, on la découvre à toutes les périodes du latin vivant, particulièrement dans le latin populaire ${ }^{29}$. Cette confusion apparaît également chez les écrivains cultivés (voir, par exemple, Sénèque, $E p ., 18,2$ ).

Voici un passage de l'historien Jordanès:

"Quis namque de Amalo dubitaret, si uacasset elegere?", Get., 174.

On remarque, dans l'apodose, l'emploi de l'imparfait de l'optatif à la place du présent et, dans la subordonnée - l'emploi du plus-que-parfait de l'optatif au lieu du parfait (le présent de l'optatif était lui aussi possible dans la conditionnelle).

À voir en plus Jordanès, Get., 164. Voir aussi le passage suivant de Fredegarii Chronicon:

"si iubebas..., accederemus ad prilium.", 2, $62{ }^{30}$, à la traduction:

"si tu donnais l'ordre, nous accéderions au combat."

27 Voir H. Goelzer, Le latin de saint Avit, op. cit., p. 357, 4.

28 Dans "C. S. E. L.", vol. VII, Vienne, 1881. La raison du choix du futur simple est, en fait, la même en latin qu'en grec ancien, voir l'opinion de J. Humbert: "En principe, le futur comporte quelque chose de plus affirmé, de plus assuré que le subjonctif", Syntaxe grecque, Paris, 1960, p. 220, par. 357 (souligné par nous)

29 Voir Ch. E. Bennett, Syntax of early Latin, I, op. cit., p. 278; voir aussi O. Riemann, Syntaxe latine d'après les principes de la grammaire historique, Paris, 1935, par. 206, Rem. 2; R. Iordache, Exprimarea ideii de conditie in limba latina, Bucuresti, "Bren", 2003, p. 88; ibid., p. 108; ibid., pp. 121-22.

${ }^{30}$ Exemple cité par M. Iliesco, dans "Istoria limbii romane" (coord. Prof. Al.Graur), vol. I, Bucarest, 1965, p. 361. Pour cette période conditionnelle, voir également le commentaire de J. Roca Melia, En torno al periodo condicional latino, dans "Volumen - homenaje al Prof. J. Campos Ruiz", Salamanca, "Kadmos", 1977, pp. 480-1 (Cf., sur cet aspect, J. B. Hofmann - A.Szantyr, Lateinische Grammatik, II -2, München, 1972, p. 662, par. 361 a). À remarquer la graphie erronée prilium (à la place de proelium). 
Saint Avit confond lui aussi, parfois, le potentiel et l'irréel - voir p. 31, 22; p. 36, 13, etc. ${ }^{31}$; voir aussi Dictys Cretensis, Ephem. Bel. Tr., 2, 23.

III. L'irréalité commence à être exprimée par des périphrases composées de "habebam + l'infinitif présent du verbe important au point de vue sémantique de l'apodose ou de la protase" (pour l'irréalité dans le présent / futur) et de "habui + l'infinitif présent du verbe de l'apodose ou de la protase" (pour exprimer l'irréalité dans le passé).

Voici un passage de Vitae Patrum:

"si uoluisset Deus ut mel manducarem, mel habuisti mittere in zippulas istas.", 5, 4,59 , à la traduction suivante:

"Si le bon Dieu avait voulu que je mange du miel, tu aurais mis du miel dans ces gâteaux." 32

\section{Autres formules périphrastiques employées dans l'apodose des périodes conditionnelles}

Les tournures périphrastiques impersonnelles des apodoses, spécifiques du latin classique, comme "oportet + 1'infinitif" et la périphrastique passive, et même les formules périphrastiques personnelles des apodoses, fréquentes dans le latin classique, disparaissent peu à peu à l'époque tardive. Cf. Cicéron:

"Si te iam, Catilina, comprehendi, si interfici iussero, credo, erit uerendum mihi ne non ....", Cat., 1, 5 .

À la basse époque, dans les apodoses apparaissent parfois des périphrases du type: "iubebor (ou iubeor à valeur de futur simple à la voix passive) accompagné de l'infinitif présent". Voir une citation de Victor Vitensis:

"Nam si hoc circa eos non fuerit obseruatum ${ }^{33}$, tam episcopus qui ordinatus fuerit uel ${ }^{34}$ clerici, sed et alii episcopi .... iubentur inter Mauros mitti.", Hist. persec., 2, 4.

Dans la citation ci-dessus iubentur est employée à la place du futur simple.

On constate aussi l'emploi dans les apodoses, à la basse époque, des périphrases composées de "iubebo / iubeo (à la voix active) ou debeo accompagnés de l'infinitif présent", exempli gratia:

"In quantum uero ut praeualet fraternitas tua, opponere se pro pauperibus, pro oppressis debet.", Gregorius Magnus, Reg., 10, 15 35.

31 Pour d'autres exemples de confusion entre le potentiel et l'irréel chez saint Avit, voir H. Goelzner, Le latin de saint Avit, op. cit., pp. 353-54.

${ }^{32} \mathrm{La}$ séquence "habui + l'infinitif présent" se trouve à la base d'une des formes de conditionnel de l'italien, voir porrebbe, saprebbe < habuit +1 'infinitif présent (forme utilisée dans la province de Toscane et dans l'italien littéraire - voir, à ce sujet, M. Savić, Temporalni kondicional u italijanskom jeziku, dans "Monografie Filološkog Fakulteta”, vol. VII, Belgrade, 1966). Voir aussi la formation du conditionnel simple en espagnol, en français etc. (<habebam + l'infinitif présent).

33 Fuerit obseruatum - séquence populaire, à la place de "erit obseruatum". Voir infra, dans le même passage, ordinatus fuerit.

$34 \mathrm{Vel}$ - au lieu de et. Pour ce qui est de la confusion entre les conjonctions copulatives et celles disjonctives dans le latin populaire de la basse époque, voir R. Iordache, Aspecte ale coordonarii in limba latina, Bucuresti, "Daim", 2003, p. 33.

35 Pour l'accumulation de connecteurs (in quantum ut), voir la discussion supra. 


\section{Cf. Pétrone:}

"si non (sc.: diligenter ponas), te iubebo in decuriam uiatorum conici.", Sat., 47, 13.

D'autres fois on observe l'emploi de l'indicatif présent ou du futur simple à valeur d'impératif (voir, par exemple, pour l'emploi du futur simple, Salvien, Gub. D., 4, 95). Il faut y ajouter l'emploi de l'impératif présent et du subjonctif présent jussif, toujours viables dans le latin populaire, pour indiquer le caractère nécessaire de l'accomplissement de l'action ou de l'événement de la principale.

\section{Aspects particuliers en ce qui concerne l'emploi des temps}

I. La prédilection du latin populaire pour l'emploi de l'indicatif présent à la place du futur simple (ou du futur antérieur), ou au lieu de l'optatif présent. Voici un passage de Pseudo-Hyginus:

"si res exigit, pedes X sufficient.", Met. castr., 9.

Le présent de l'indicatif est agréé non seulement dans les subordonnées, naais encore dans les régissantes - voir Pseudo-Hyginus, Met. castr., 18; Victor Vitensis, Hist. persec., 2, 4, etc.

Dans les conditionnelles apparait souvent le présent de l'indicatif, pour indiquer "le caractère immédiat de la condition" 36 . Exempli gratia:

- "Quomodo uero Getae Gepidasque ${ }^{37}$ sint parentes si quaeris, paucis absoluam.", Jordanès, Get., 94.

- "Ille uero animo pretumido ${ }^{38}$ ait: 'Si hic ${ }^{39}$ murmuras et me uenire causaris, Tolosam, ubi tu sedes, ueniam.' ...", Jordanès, Get., 231.

II. L'emploi du plus-que-parfait du subjonctif (optatif) à la place de l'imparfait du subjonctif (optatif). Le plus-que-parfait du subjonctif remplace fréquemment, à l'époque tardive, l'imparfait du même mode, dans des propositions principales et également dans des subordonnées de types différents (propositions relatives, comparatives, consécutives, finales, temporelles, conditionnelles, complétives). Il s'agit d'un phénomène important du latin populaire, déjà amplement attesté dans le Bellum Africum ${ }^{40}$. À la basse époque, les attestations de cet emploi sont de plus en plus nombreuses ${ }^{41}$.

${ }^{36}$ Quant à l'emploi du présent de l'indicatif pour indiquer "le caractère immédiat de la condition", voir A. Ernout - Fr. Thomas, Syntaxe latine, Paris, 1989, pp. 375-6.

${ }^{37}$ Gepidas - forme de nominatif pluriel, assez répandue chez Jordanès (correspondant au nominatif gothique - voir, sur ce sujet, la note de Th. Mommsen, Iordanis Romana et Getica, Berlin, 1961, p. 160).

38 Pretumido - graphie populaire (au lieu de praetumido).

${ }^{39} \mathrm{Hic}$ - adverbe, ici à fonction de complément de propos (à la place de la séquence: "de hac re").

40 Voir Wilh. Meyer-Lùbke, Die lateinische Sprache in den romanischen Ländern, dans "Grundriss Gröber", I, p. 451 sqq.

41 Voir H. Rönsch, Itala und Vulgata, das Sprachidiom der urchristlichen Itala und der katolischen Vulgata unter Berücksichtigung der römischen Volkssprache, Marburg, 1875, p. 431; C. H. Grandgent, Introducción al latinvulgar, Madrid, 1928, p. 95: A. Blaise, Manuel du latin chrétien, op. cit., p. 136; etc. 
Voici quelques exemples témoignant de cet emploi du plus-que-parfait du subjonctif:

- "Nam si haec praesens uita sola fuisset et aliam, quae uere est, non speraremus aeternam, nec ita fecissem ....", Victor Vitensis, Hist. persec., 3, 27.

- "Nam si nunc superessent uel eis fari de talibus rebus licuisset, et Tullianae eloquentiae fluuius siccaretur, et Sallustius elinguis omnimodis remaneret.", Victor Vitensis, Hist. persec., 3, 61 42.

Chez certains auteurs tardifs, l'imparfait du subjonctif est employé à la place du plus-que-parfait du subjonctif. Il s'agit de hyperurbanismes. Exempli gratia:

"... et pene Attilam trucidarent, nisi prouidus prius fugisset et se suosque ilico intra septa ${ }^{43}$ castrorum.... reclusisset.", Jordanès, Get., 210.

Voir également le passage cité - Victor Vitensis, Hist. persec., 1, 27; ibid., 1, 18.

Ce phénomène (l'emploi de l'imparfait du subjonctif au lieu du plus-que-parfait $\mathrm{du}$ subjonctif) reste rare à la basse époque ${ }^{44}$.

En Résumé, la subordonnée conditionnelle, fréquemment employée à la basse époque, dans le registre populaire, présente certaines particularités. Pour ce qui est des modes et des temps on peut remarquer:

I. Un usage élargi de l'indicatif à la place de l'optatif, aussi bien dans les subordonnées que dans les régissantes. Il s'agit de l'usage de l'indicatif présent et même du futur simple en remplacement de l'optatif présent; de l'emploi des temps passés de l'indicatif pour exprimer l'irréalité.

II. La confusion (qui est d'ailleurs constante dans le latin populaire, à partir de l'époque préclassique) du potentiel et de l'irréel, confusion présent tantôt dans des subordonnées, tantôt dans des régissantes. Nous faisons référence ici aux fautes dues à l'ignorance, à la hâte, et non pas au changement des temps pour des raisons stylistiques (phénomène fréquent dans les prosopopées), ou pour la mise en pratique des règles de la concordance des temps, ou bien pour des raisons de métrique.

III. L'irréalité commence à être exprimée en périphrases formées de "habebam + infinitif présent" (pour l'irréalité dans le présent / futur) et de "habui + infinitif présent" (pour l'irréalité dans le passé). Ces périphrases sont employées aussi bien dans les protases que dans les apodoses.

IV. La prédilection du latin populaire pour l'utilisation de l'indicatif présent à la place du futur simple (ou du futur antérieur), ou à la place de l'optatif présent dans les protases.

V. L'emploi du plus-que-parfait du subjonctif (optatif) à la place de l'imparfait du subjonctif (optatif), dans les protases et également dans les apodoses.

42 Exemples tirés de l'édition de M. Petschenig, Victoris Episcopi Vitensis 'Historia persecutionis Africanae prouinciae', Index uerborum et locutionum, dans "C. S. E. L.", VII, Vienne, 1881, p. 167.

43 On remarque, dans cette citation, les graphies populaires: septa (àla place de saepta) et pene (au lieu de paene).

44 Voir D. Norberg, Manuel pratique de latin médiéval, Paris, 1968, p. 171; C. H. Grandgent, Introducción al latin vulgar, op. cit., p. 95. Cf. H. Rönsch, Itala und Vulgata, op. cit., p. 431. 
En ce qui concerne l'inventaire de conjonctions et de locutions, à part l'usage de $s i$ et si non (panromanes), le latin populaire de la basse époque emploie volontiers: siue, avec la variante seu, et même nisi, ainsi que certaines locutions conjonctives ayant pour base la conjonction si (si forte, si quidem, sane si, plane si, si tantum, si tamen, si uero, si contra, e contra si). Pour la disjonction entre deux propositions conditionnelles, les auteurs tardifs employaient tantôt siue (seu), tantôt aut, ou aut si. Avec le sens de "pourvu que" est utilisée la conjonction tantum ou des locutions telles que: "tantum ut", "tantum ne / non", "sub hac condicione", "cum hac condicione", ou bien "cum hoc", "in hoc", accompagnées de propositions à fonction d'apposition, introduites par ut / quod, si.

Il y a encore d'autres conjonctions et locutions populaires qui apparaissent parfois dans un contexte conditionnel: quando, ubi, dum, in quantum, in quo.

Les langues romanes ont puisé dans le latin populaire de la basse époque des conjonctions, des locutions conjonctives, des corrélatifs, certaines constructions grammaticales, y compris des syntagmes exprimant le potentiel et l'irréel (parfois différents d'un idiome àl'autre).

\section{Povzetek \\ POGOJNI ODVISNIK LJUDSKE RABE V POZNI LATINŠČINI}

Pogojni odvisnik, ki se je $v$ pozni dobi pogosto uporabljal $v$ ljudskem registru, kaže določene posebnosti z ozirom na kultivirano latinščino klasične dobe. Glede naklonov in časov lahko pripomnimo:

I. Razširjena raba indikativa namesto optativa tako v odvisnih kot v glavnih stavkih.

II. Zmeda (ta je v ljudski latinščini sicer staina od predklasične dobe) potencialnosti in irealnosti, ki se pojavlja tako v odvisnih kot v glavnih stavkih.

III. Irealnost se začne izražati z opisnimi zvezami habebam + infinitiv prezenta (za pretekli irrealis). Te opisne zveze se uporabljajo tako $v$ odvisnih kot $v$ glavnih stavkih.

IV. Nagnjenje ljudske latinščine $\mathrm{k}$ rabi indikativa prezenta namesto indikativa futura (ali futura II) oz. namesto optativa prezenta $\mathrm{v}$ odvisniku.

$\mathrm{V}$. Raba optativa pluskvamperfekta namesto optativa imperfekta tako $\mathrm{v}$ odvisnih kot $\mathrm{v}$ glavnih stavkih.

Kar zadeva inventar veznikov in stalnih zvez, začenši z rabo si in si non (vseromansko) ljudska latinščina rada uporablja: sive z inačico seu, celo nisi, kot tudi določene zveze, ki temeljijo na vezniku si (si forte, si quidem, sane si, plane si, si tantum idr.). s pomenom 'pod pogojem, da' se uporablja veznik tantum ali zveze kot tantum ut, tantum ne/non, sub hac condicione, cum hac condicione ali cum hoc, in hoc, ki jih spremljajo stavki v vlogi apozicije, uvedeni $z$ ut/quod si.

Včasih se v pogojni zvezi pojavijo še drugi vezniki in ljudske zveze: quando, ubi, dum, in quantum, in quo.

Romanski jeziki so črpali iz ljudske latinščine pozne dobe veznike, pogojne zveze, soodnosnice, določene slovnične konstrukcije vključno z zvezami, ki izražajo potencialnost in irealnost (včasih od jezika do jezika različne). 